\title{
A Hybrid Optimization Technique Coupling an Evolutionary and a Local Search Algorithm for Economic Emission Load Dispatch Problem
}

\author{
A. A. Mousa, Kotb A. Kotb \\ Department of Mathematics and Statistics, Faculty of sciences, Taif University, Taif, \\ El-Haweiah, Kingdom of Saudi Arabia (KSA) \\ E-mail:a_mousa15@yahoo.com \\ Received May 13, 2011; revised May 24, 2011; accepted May 27, 2011
}

\begin{abstract}
This paper presents an optimization technique coupling two optimization techniques for solving Economic Emission Load Dispatch Optimization Problem EELD. The proposed approach integrates the merits of both genetic algorithm (GA) and local search (LS), where it maintains a finite-sized archive of non-dominated solutions which gets iteratively updated in the presence of new solutions based on the concept of $\varepsilon$-dominance. To improve the solution quality, local search technique was applied as neighborhood search engine, where it intends to explore the less-crowded area in the current archive to possibly obtain more nondominated solutions. TOPSIS technique can incorporate relative weights of criterion importance, which has been implemented to identify best compromise solution, which will satisfy the different goals to some extent. Several optimization runs of the proposed approach are carried out on the standard IEEE 30-bus 6-genrator test system. The comparison demonstrates the superiority of the proposed approach and confirms its potential to solve the multiobjective EELD problem.
\end{abstract}

Keywords: Economic Emission Load Dispatch, Evolutionary Algorithms, Multiobjective Optimization, Local Search

\section{Introduction}

The purpose of EELD problem is to figure out the optimal amount of the generated power for the fossil-based generating units in the system by minimizing the fuel cost and emission level simultaneously, subject to various equality and inequality constraints including the security measures of the power transmission/distribution.

Different techniques have been reported in the literature pertaining to economic emission load dispatch problem. In $[1,2]$ the problem has been reduced to a single objective problem by treating the emission as a constraint with a permissible limit. This formulation, however, has a severe difficulty in getting the trade-off relations between cost and emission. Alternatively, minimizing the emission has been handled as another objective in addition to usual cost objective. A linear programming based optimization procedures in which the objectives are considered one at a time was presented in [3]. Unfortunately, the EELD problem is a highly nonlinear and a multimodal optimization problem. Therefore, conventional optimization methods that make use of derivatives and gradients, in general, not able to locate or identify the global optimum. On the other hand, many mathematical assumptions such as analytic and differential objective functions have to be given to simplify the problem. Furthermore, this approach does not give any information regarding the trade-offs involved.

In other research direction, the multiobjective EELD problem was converted to a single objective problem by linear combination of different objectives as a weighted sum [4-7]. The important aspect of this weighted sum method is that a set of Pareto-optimal solutions can be obtained by varying the weights. Unfortunately, this requires multiple runs as many times as the number of desired Pareto-optimal solutions. Furthermore, this method cannot be used to find Pareto-optimal solutions in problems having a nonconvex Pareto-optimal front. In addition, there is no rational basis of determining adequate weights and the objective function so formed may lose 
significance due to combining noncommensurable objectives. To avoid this difficulty, the $\varepsilon$-constraint method for multiobjective optimization was presented in $[8,9]$. This method is based on optimization of the most preferred objective and considering the other objectives as constraints bounded by some allowable levels. These levels are then altered to generate the entire Pareto-optimal set. The most obvious weaknesses of this approach are that it is time-consuming and tends to find weakly nondominated solutions.

Goal programming method was also proposed for multiobjective EELD problem [10]. In this method, a target or a goal to be achieved for each objective is assigned and the objective function will then try to minimize the distance from the targets to the objectives. Although the method is computationally efficient, it will yield an inferior solution rather than a noninferior one if the goal point is chosen in the feasible domain. Hence, the main drawback of this method is that it requires a priori knowledge about the shape of the problem search space.

Heuristic algorithms such as genetic algorithm have been recently proposed for solving OPF problem [11-13]. The results reported were promising and encouraging for further research. Moreover the studies on heuristic algorithms over the past few years, have shown that these methods can be efficiently used to eliminate most of difficulties of classical methods [14-18]. Since they are population-based techniques, multiple Pareto-optimal solutions can, in principle, be found in one single run.

In this paper a hybrid multiobjective approach is proposed, which based on concept of co-evolution and repair algorithm for handing constraints. $\varepsilon$-Dominance concept was implemented to maintains a finite-sized archive of non-dominated solutions which gets iteratively updated according to the chosen $\varepsilon$-vector. TOPSIS [19] approach has been implemented to select best compromise solution, which will satisfy the different goals to some extent. Also, LS method was introduced as neighborhood search engine where it intends to explore the less-crowded area in the current archive to possibly obtain more nondominated solutions.

\section{Multiobjective Optimization}

Multiobjective optimization [11] differs from the single objective case in several ways:

- The usual meaning of the optimum makes no sense in the multiple objective case because the solution optimizing all objectives simultaneously is, in general, impractical; instead, a search is launched for a feasible solution yielding the best compromise among objectives on a set of, so called, efficient solutions;

- The identification of a best compromise solution requires taking into account the preferences expressed by the decision-maker;

- The multiple objectives encountered in real-life problems are often mathematical functions of contrasting forms.

- A key element of a goal programming model is the achievement function; that is, the function that measures the degree of minimization of the unwanted deviation variables of the goals considered in the model. A general multiobjective optimization problem is expressed by:

$$
\begin{aligned}
\min F(x)= & \left(f_{1}(x), f_{2}(x), \cdots, f_{m}(x)\right)^{\mathrm{T}} \\
& \text { s.t. } \quad x \in S \\
x= & \left(x_{1}, x_{2}, \cdots, x_{n}\right)^{\mathrm{T}}
\end{aligned}
$$

where $f_{1}(x), f_{2}(x), \cdots, f_{m}(x)$ are the $m$ objectives functions, $\left(x_{1}, x_{2}, \cdots, x_{n}\right)$ are the $n$ optimization parameters, and $S \in R^{n}$ is the solution or parameter space.

Definition 1. (Pareto optimal solution ): $x^{*}$ is said to be a Pareto optimal solution of MOP if there exists no other feasible $x$ (i.e., $x \in S$ ) such that, $f_{j}(x) \leq f_{j}\left(x^{*}\right)$ for all $j=1,2, \cdots, m$ and $f_{j}(x)<f_{j}\left(x^{*}\right)$ for at least one objective function $f_{j}$.

Definition 2 [20]. ( $\varepsilon$-dominance) Let $f: x \rightarrow R^{m}$ and $a, b \in X$. Then $a$ is said to $\varepsilon$-dominate $b$ for some $\varepsilon$ $>0$, denoted as $a \succ_{\varepsilon} b$, if and only if for $i \in\{1, \cdots, m\}$ $(1-\varepsilon) f_{i}(a) \leq f_{i}(b)$

According to Definition 2, the $\varepsilon$ value stands for a relative "tolerance" allowed for the objective values which declared in Figure 1. This is especially important in higher dimensional objective spaces, where the concept of $\varepsilon$-dominance can reduce the required number of solutions considerably. Also, the use of $\varepsilon$-dominance also makes the algorithms practical by allowing a decision maker to control the resolution of the Pareto set approximation by choosing an appropriate $\varepsilon$ value.

\section{Economic Emission Load Dispatch (EELD)}

The economic emission load dispatch involves the simultaneous optimization of fuel cost and emission object-

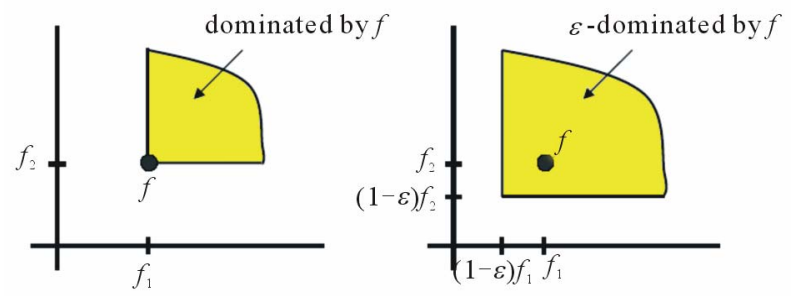

Figure 1. Graphs visualizing the concepts of dominance (left) and $\varepsilon$-dominance (right). 
tives which are conflicting ones. The deterministic problem is formulated as described below.

\subsection{Objective Functions}

Fuel Cost Objective. The classical economic dispatch problem of finding the optimal combination of power generation, which minimizes the total fuel cost while satisfying the total required demand can be mathematically stated as follows [9]:

$$
f(\cdot)=C_{t}=\sum_{i=1}^{n} C_{i}\left(P_{G i}\right)=\sum_{i=1}^{n}\left(a_{i}+b_{i} P_{G i}+c_{i} P_{G i}^{2}\right) \$ / \mathrm{hr}
$$

where

$C$ : total fuel cost $(\$ / \mathrm{hr}), C_{i}$ : is fuel cost of generator $i$

$a_{i}, b_{i}, c_{i}$ : fuel cost coefficients of generator $i$,

$P_{G i}$ : power generated ( $\left.p . u\right)$ by generator $i$,

$n$ : number of generator.

Emission Objective. The emission function can be presented as the sum of all types of emission considered, such as $\mathrm{NO}_{x}, \mathrm{SO}_{2}$, thermal emission, etc., with suitable pricing or weighting on each pollutant emitted. In the present study, only one type of emission $\mathrm{NO}_{x}$ is taken into account without loss of generality. The amount of $\mathrm{NO}_{x}$ emission is given as a function of generator output, that is, the sum of a quadratic and exponential function:

$$
\begin{aligned}
& f_{2}(\cdot)=E_{N O_{x}} \\
& =\sum_{i=1}^{n}\left[10^{-2}\left(\alpha_{i}+\beta_{i} P_{G i}+\gamma_{i} P_{G i}^{2}\right)+\xi_{i} \exp \left(\lambda_{i} P_{G i}\right)\right] \text { ton } / \mathrm{hr}
\end{aligned}
$$

where, $\alpha_{i}, \beta_{i}, \gamma_{i}, \xi_{i}, \lambda_{i}$ : coefficients of the $i$ th generator's $\mathrm{NO}_{x}$ emission characteristic.

\subsection{Constraints}

The optimization problem is bounded by the following constraints:

- Power balance constraint. The total power generated must supply the total load demand and the transmission losses.

$$
\sum_{i=1}^{n} P_{G i}-P_{D}-P_{\text {Loss }}=0
$$

where $P_{D}$ : total load demand (p.u.), and $P_{\text {loss }}$ : transmission losses (p.u.).

The transmission losses are given by [21]:

$$
P_{\text {Loss }}=\sum_{i=1}^{n} \sum_{i=1}^{n}\left[A_{i j}\left(P_{i} P_{j}+Q_{i} Q_{j}\right)+B_{i j}\left(Q_{i} P_{j}-P_{i} Q_{j}\right)\right]
$$

where

$$
\begin{aligned}
& P_{i}=P_{G i}-P_{D i}, \quad \mathrm{Q}_{i}=Q_{G i}-Q_{D i}, \\
& \mathrm{~A}_{i j}=\frac{R_{i j}}{V_{i} V_{j}} \cos \left(\delta_{i}-\delta_{j}\right), \quad \mathrm{B}_{i j}=\frac{R_{i j}}{V_{i} V_{j}} \sin \left(\delta_{i}-\delta_{j}\right)
\end{aligned}
$$

$n$ : number of buses

$R_{i j}$ : series resistance connecting buses $i$ and $j$

$V_{i}$ : voltage magnitude at bus $i$

$\delta_{i}$ : voltage angle at bus $i$

$P_{i}$ : real power injection at bus $i$

$Q$ : reactive power injection at bus $i$

- Maximum and Minimum Limits of Power Generation. The power generated $P_{G i}$ by each generator is constrained between its minimum and maximum limits, i.e.,

$$
\begin{aligned}
& P_{G i \min } \leq P_{G i} \leq P_{G i \max }, \quad Q_{G i \min } \leq Q_{G i} \leq Q_{G i \max }, \\
& V_{i \text { min }} \leq V_{i} \leq V_{i \max }, \quad i=1, \cdots, n
\end{aligned}
$$

where $P_{G i \min }$ : minimum power generated, and $P_{G i \max }$ : maximum power generated.

- Security Constraints. A mathematical formulation of the security constrained EELD problem would require a very large number of constraints to be considered. However, for typical systems the large proportion of lines has a rather small possibility of becoming overloaded. The EELD problem should consider only the small proportion of lines in violation, or near violation of their respective security limits which are identified as the critical lines. We consider only the critical lines that are binding in the optimal solution. The detection of the critical lines is assumed done by the experiences of the DM. An improvement in the security can be obtained by minimizing the following objective function.

$$
S=f\left(P_{G i}\right)=\sum_{j=1}^{k}\left(\left|T_{j}\left(P_{G}\right)\right| / T_{j}^{\max }\right)
$$

where, $T_{j}\left(P_{G}\right)$ is the real power flow $T_{j}^{\max }$ is the maximum limit of the real power flow of the $j$ th line and $k$ is the number of monitored lines. The line flow of the $j$ th line is expressed in terms of the control variables $P_{G s}$, by utilizing the generalized generation distribution factors (GGDF) [22] and is given below.

$$
T_{J}\left(P_{G}\right)=\sum_{i=1}^{n}\left(D_{j i} P_{G i}\right)
$$

where, $D_{j i}$ is the generalized GGDF for line $j$, due to generator $i$.

For secure operation, the transmission line loading $S_{l}$ is restricted by its upper limit as

$$
S_{\ell} \leq S_{\ell \max }, \ell=1, \cdots, n_{\ell}
$$

where $n_{\ell}$ is the number of transmission line.

\subsection{Multiobjective Formulation of EELD Problem}

The multiobjective EELD optimization problem [11] is 
therefore formulated as:

$$
\begin{aligned}
& \text { Min } f_{1}(x)=C_{t} \\
& =\sum_{i=1}^{n}\left(a_{i}+b_{i} P_{G i}+c_{i} P_{G i}^{2}\right) \$ / h r \\
& \operatorname{Min} f_{2}(\cdot)=E_{N O_{x}} \\
& =\sum_{i=1}^{n}\left[10^{-2}\left(\alpha_{i}+\beta_{i} P_{G i}+\gamma_{i} P_{G i}^{2}\right)+\xi_{i} \exp \left(\lambda_{i} P_{G i}\right)\right] \text { ton } / h r \\
& \text { s.t. } \quad \sum_{i=1}^{n} P_{G i}-P_{D}-P_{\text {Loss }}=0 \text {, } \\
& S_{\ell} \leq S_{\ell \max }, \\
& P_{G i \min } \leq P_{G i} \leq P_{G i \max } \\
& \ell=1, \cdots, n_{\text {Line }}, \\
& Q_{G i \min } \leq Q_{G i} \leq Q_{G i \max } \\
& i=1, \cdots, n \\
& V_{i \min } \leq V_{i} \leq V_{i \max } \\
& i=1, \cdots, n \\
& i=1, \cdots, n
\end{aligned}
$$

\section{The Proposed Algorithm}

Recently, the studies on evolutionary algorithms have shown that these algorithms can be efficiently used to eliminate most of the difficulties of classical methods which can be summarized as:

- An algorithm has to be applied many times to find multiple Pareto-optimal solutions.

- Most algorithms demand some knowledge about the problem being solved.

- Some algorithms are sensitive to the shape of the Pareto-optimal front.

- The spread of Pareto-optimal solutions depends on efficiency of the single objective optimizer.

It is worth mentioning that the goal of a multiobjective optimization problem is not only guide the search towards Pareto-optimal front but also maintain population diversity.

\subsection{Initialization Stage}

The algorithm uses two separate population [11], the first population $P^{(t)}$ consists of the individuals which initialized randomly satisfying the search space (The lower and upper bounds), while the second population $R^{(t)}$ consists of reference points which satisfying all constraints. However, in order to ensure convergence to the true Pareto-optimal solutions, we concentrated on how elitism could be introduced in the algorithm. So, we propose an archiving/selection [20] strategy that guarantees at the same time progress towards the Pareto-optimal set and a covering of the whole range of the non-dominated solutions. The algorithm maintains an externally finite-sized archive $A^{(t)}$ of non-dominated solutions which gets iteratively updated in the presence of new solutions based on the concept of $\varepsilon$-dominance.

\subsection{Repair Algorithm}

The idea of this technique [11] is to separate any feasible individuals in a population from those that are infeasible by repairing infeasible individuals. This approach coevolves the population of infeasible individuals until they become feasible. Repair process works as follows. Assume, there is a search point $\omega \notin S$ (where $S$ is the feasible space). In such a case the algorithm selects one of the reference points (Better reference point has better chances to be selected), say $r \in S$ and creates random points $\bar{Z}$ from the segment defined between $\omega, r$, but the segment may be extended equally on both sides determined by a user specified parameter $\mu \in[0,1]$. Thus, a new feasible individual is expressed as:

$$
z_{1}=\gamma \cdot \omega+(1-\gamma) \cdot r, \quad z_{2}=(1-\gamma) \cdot \omega+\gamma \cdot r
$$

where $\gamma=(1+2 \mu) \delta-\mu$ and $\delta \in[0,1]$ is a random generated number

\subsection{LS Stage}

In this stage, we present modified local search technique (MLS) [23] to improve the solution quality and to explore the less-crowded area in the external archive to possibly obtain more nondominated solutions nearby. We propose a MLS, which is a modification of Hooke and Jeeves method [24] to be suitable for MOP. The general procedure of the MLS techniques can be described by the following steps.

Step 1. Start with an arbitrarily chosen point $\left(X_{m} \in \mathbb{R}^{n}\right) \in E^{t}$, and the prescribed step lengths $\Delta x_{i}$ in each of the coordinate directions $u_{i}, i=1,2, \cdots, n$. Set $m$ $=0$, assume that $m$ is the size of $E^{t}$.

Step 2. Set $m=m+1$, and $k=1$ where $k$ is number of trial (s.t., $k=1, \cdots, k_{\max }$ ) to obtain preferred solution than $X_{m}$.

Step 3. The variable $x_{i}$ is perturbed about the current temporary base point $X_{m}$ to obtain the new temporary base point $X_{m}^{\prime}$ as :

$$
\begin{aligned}
& X_{m}^{\prime}= \\
& \left\{\begin{array}{l}
X_{m}+\Delta x_{i} u_{i} \text { if } f^{+}(\cdot) \succ f \\
X_{m}-\Delta x_{i} u_{i} \text { if } f^{-}(\cdot) \succ\left(f(\cdot) \wedge f^{+}(\cdot)\right) \quad \forall i=1,2, \cdots, n \\
X_{m} \quad \text { if } f(\cdot) \succ\left(f^{+}(\cdot) \wedge f^{-}(\cdot)\right)
\end{array}\right.
\end{aligned}
$$

where, $f(\cdot)=f\left(X_{m}\right), f^{+}(\cdot)=f\left(X_{m}+\Delta x_{i} u_{i}\right)$, and $f^{-}(\cdot)=f\left(X_{m}-\Delta x_{i} u_{i}\right)$. Assume $f(\cdot)$ is the evaluation of the objective functions at a point.

Step 4. If the point $X_{m}$ unchanged.

While the number of trial $k$ not satisfied, reduce the step length $\Delta x_{i}$. The following dynamic equation is presented to reduce $\Delta x_{i}$, 


$$
\Delta x_{i}=\Delta x_{i}\left(1-(r)^{\frac{k}{k_{\max }}}\right), \quad r \in[0,1]
$$

and go to step 3 .

Step 5. Else, if $X_{m}^{\prime}$ is preferred than $X_{m}$ (i.e., $\left.f\left(X_{m}^{\prime}\right) \succ f\left(X_{m}\right)\right)$, then $X_{m}^{\prime}$ is the new base point.

Step 6. With the help of the base points $X_{m}$ and $X_{m}^{\prime}$, establish a pattern direction $\mathrm{S}$ as

$$
S=X_{m}^{\prime}-X_{m}
$$

and find a point $X_{m}^{\prime \prime}$ as $X_{m}^{\prime \prime}=X_{m}^{\prime}+\lambda \mathrm{S}$, where $\lambda$ is the step length, (taken as 1).

Step 7. If $f\left(X_{m}^{\prime \prime}\right) \succ f\left(X_{m}^{\prime}\right)$ set $X_{m}=X_{m}^{\prime}, X_{m}^{\prime}=X_{m}^{\prime \prime}$, and go to 6 . 4.

Step 8. If $f\left(X_{m}^{\prime \prime}\right) \nsucc f\left(X_{m}^{\prime}\right)$ set $X_{m}=X_{m}^{\prime}$, and go to

These steps is implemented on all nondominated solutions in $A^{t}$ to get the true Pareto optimal solution and to explore the less-crowded area in the external archive. Figure 2 shows the pseudo code of the MLS algorithm.

\subsection{Identifying a Best Compromise Solution}

Optimization of the above-formulated objective functions [11] yields not a single optimal solution, but a set of Pareto optimal solutions, in which one objective cannot be improved without sacrificing other objectives. For practical applications, however, we need to select one solution, which will satisfy the different goals to some extent. Such a solution is called best compromise solution. TOPSIS method given by Yoon and Hwang [19] has the ability to identify the best alternative from a finite set of alternatives quickly. It stands for "Technique for Order Preference by Similarity to the Ideal Solution" which based upon the concept that the chosen alternative should have the shortest distance from the positive ideal solution and the farthest from the negative ideal solution.

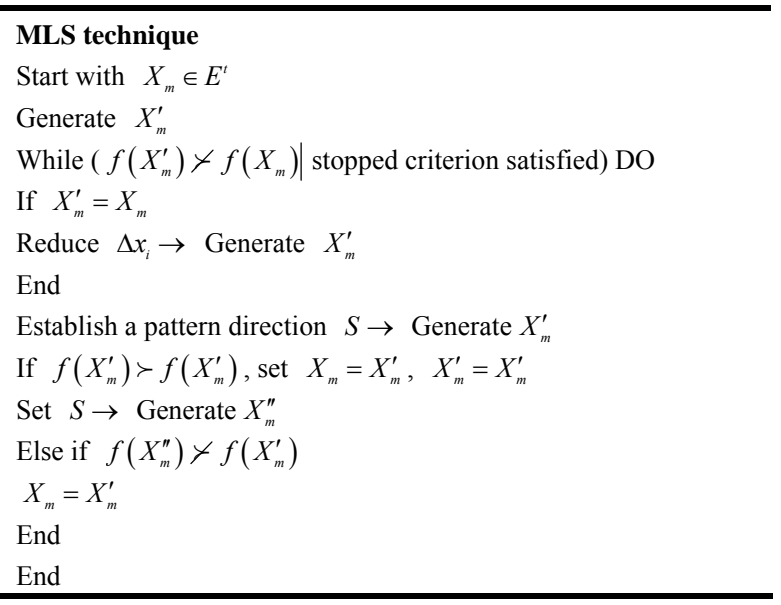

Figure 2. The pseudo code of the MLS algorithm.
TOPSIS can incorporate relative weights of criterion importance. The idea of TOPSIS can be expressed in a series of steps.

Step1: Obtain performance data for $n$ alternatives over $M$ criteria $x_{i j}(i=1, \cdots, n, j=1, \cdots, M)$.

Step2: Calculate normalized rating (vector normalization is used) $r_{i j}$.

Step3: Develop a set of importance weights $W_{j}$, for each of the criteria. The basis for these weights can be anything, but, usually, is adhoc reflective of relative importance.

$$
V_{i j}=w_{j} \cdot r_{i j}
$$

Step4: Identify the ideal alternative (extreme performance on each criterion) $S^{+}$.

$$
\begin{aligned}
S^{+} & =\left\{v_{1}^{+}, \cdots, v_{j}^{+}, \cdots, v_{m}^{+}\right\} \\
& =\left\{\left(\max v_{i j} \mid j \in J_{1}\right),\left(\min v_{i j} \mid j \in J_{2}\right), i=1, \cdots, n\right\}
\end{aligned}
$$

where $J_{!}$is a set of benefit attributes and $J_{2}$ is a set of cost attributes.

Step5: Identify the nadir alternative (reverse extreme performance on each criterion) $S^{-}$.

$$
\begin{aligned}
S^{-} & =\left\{v_{1}^{-}, \cdots, v_{j}^{-}, \cdots, v_{m}^{-}\right\} \\
& =\left\{\left(\min v_{i j} \mid j \in J_{1}\right),\left(\max v_{i j} \mid j \in J_{2}\right), i=1, \cdots, n\right\}
\end{aligned}
$$

Step6: Develop a distance measure over each criterion to both ideal $\left(D^{+}\right)$and nadir $\left(D^{-}\right)$.

$$
D_{i}^{+}=\sqrt{\sum_{j}\left(v_{i j}-v_{j}^{+}\right)^{2}}, \quad D_{i}^{-}=\sqrt{\sum_{j}\left(v_{i j}-v_{j}^{-}\right)^{2}}
$$

Step7: For each alternative, determine a ratio $R$ equal to the distance to the nadir divided by the sum of the distance to the nadir and the distance to the ideal,

$$
R=\frac{D^{-}}{D^{-}+D^{+}}
$$

Step8: Rank alternative according to ratio $R$ (in Step 7) in descending order, recommend the alternative with the maximum ratio. The pseudo code of the proposed algorithm are shown in Figure 3.

\subsection{Basic Algorithm}

It uses two separate population, the first population $P^{(t=0)}$ (where $t$ is the iteration counter) consists of the individuals which initialized randomly satisfying the search space, while the second population $R^{(t)}$ consists of reference points which satisfying all constraints. Also, it stores initially the Pareto-optimal solutions externally in a finite sized archive of non-dominated solutions $A^{(0)}$. We use cluster algorithm to create the next population 


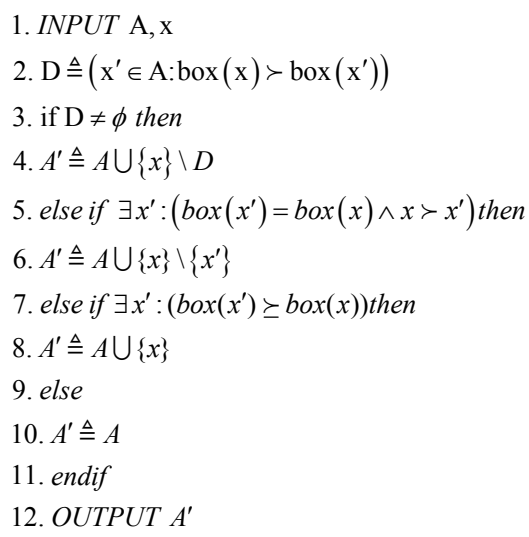

Figure 3. Algorithm of select operator.

$P^{(t+1)}$, if $\left|P^{(t)}\right|>\left|A^{(t)}\right|$ (i.e., the size of the population $P^{(t)}$ greater than the size of archive $\boldsymbol{A}^{(t)}$ ) then new population $P^{(t+1)}$ consists of all individual from $A^{(t)}$ and the population $P^{(t)}$ are considered for the clustering procedure to complete $P^{(t+1)}$, if $\left|P^{(t)}\right|<\left|A^{(t)}\right|$ then $|P|$ solutions are picked up at random from $A^{(t)}$ and directly copied to the new population $P^{(t+1)}$.

Since our goal is to find new nondominated solutions, one simple way to combine multiple objective functions into a scalar fitness function is the following weighted sum approach:

$$
f(x)=w_{1} f_{1}(x)+\cdots+w_{m} f_{m}(x)=\sum_{j=1}^{m} w_{j} f_{j}(x)
$$

where $x$ is a string (i.e., individual), $f(x)$ is a combined fitness function, $f_{i}(x)$ is the ith objective function. When a pair of strings is selected for a crossover operation, we assign a random number to each weight as follows.

$$
w_{i}=\frac{\operatorname{random}_{i}(.)}{\sum_{j=1}^{m} \operatorname{random}_{j}(.)}, \quad i=1,2, \cdots, m
$$

Calculate the fitness value of each string using the random weights $w_{i}$. Select a pair of strings from the current population according to he following selection probability $\beta(x)$ of a string $\mathrm{x}$ in the population $P^{(t)}$

$$
\beta(x)=\frac{f(x)-f_{\min }\left(P^{(t)}\right)}{\sum_{x \in P^{(t)}}\left\{f(x)-f_{\min }\left(P^{(t)}\right)\right\}},
$$

where $f_{\min }\left(P^{(t)}\right)=\min \left\{f(x) \mid x \in P^{(t)}\right\}$

This step is repeated for selecting $|P| / 2$ Paris of strings from the current populations. For each selected pair apply crossover operation to generate two new strings, for each strings generated by crossover operation, apply a mutation operator with a pre-specified mutation prob- ability. The system also includes the survival of some of the good individuals without crossover or selection. This method seems to be better than the others if applied constantly.

Algorithm in Figure 4, shows the proposed algorithm. The purpose of the function generate is to generate a new population in each iteration $t$, possibly using the contents of the old population $P^{(t-1)}$ and the old archive set $A^{(t-1)}$ in associated with variation (recombination and mutation). The function update gets the new population $P^{(t)}$ and the old archive set $A^{(t-1)}$ and determines the updated one, namely $A^{(t)}$ as indicated in Figure 3. The function $\boldsymbol{L s}$ is to explore the less-crowded area in the current archive to possibly obtain more nondominated solutions which declared in pseudo code in Figure 2.

The algorithm maintains a finite-sized archive of nondominated solutions which gets iteratively updated in the presence of a new solutions based on the concept of $\varepsilon$-dominance, such that new solutions are only accepted in the archive if they are not $\varepsilon$-dominated by any other element in the current archive (Figure 3), The use of $\varepsilon$-dominance also makes the algorithms practical by allowing a decision maker to control the resolution of the Pareto set approximation by choosing an appropriate $\varepsilon$ value.

\section{Implementation of the Proposed Approach}

The described methodology is applied to the standard IEEE 30-bus 6-generator test system to investigate the effectiveness of the proposed approach. The values of fuel cost and emission coefficients are given in Table $\mathbf{1 .}$ For comparison purposes with the reported results, the system is considered as losses and the security constraint is released. The techniques used in this study were developed and implemented on 1.7-MHz PC using MATLAB environment. Table 2 lists the parameter setting used in the algorithm for all runs.

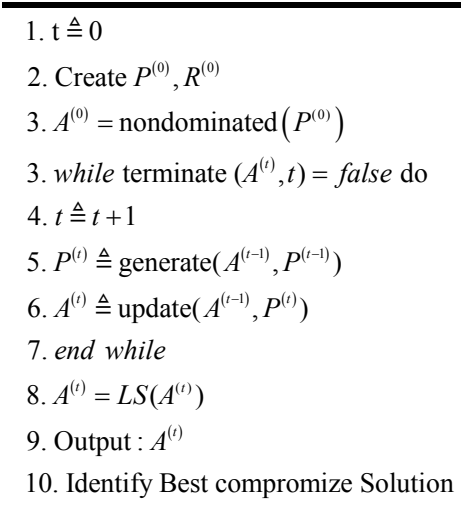

Figure 4. Algorithm of the proposed algorithm. 
Table 1. Generator cost and emission coefficients.

\begin{tabular}{cccccccc}
\hline & & G1 & G2 & G3 & G4 & G5 & G6 \\
\hline Cost & a & 10 & 10 & 20 & 10 & 20 & 10 \\
& b & 200 & 150 & 180 & 100 & 180 & 150 \\
& c & 100 & 120 & 40 & 60 & 40 & 100 \\
Emission & $\alpha$ & 4.09 & 2.54 & 4.25 & 5.42 & 4.25 & 6.13 \\
& $\beta$ & -5.55 & -6.04 & -5.09 & -3.55 & -5.09 & -5.55 \\
& $\gamma$ & 6.49 & 4.63 & 4.58 & 3.38 & 4.58 & 5.15 \\
& $\zeta$ & $2 \mathrm{E}-4$ & $5 \mathrm{E}-4$ & $1 \mathrm{E}-6$ & $2 \mathrm{E}-3$ & $1 \mathrm{E}-6$ & $1 \mathrm{E}-5$ \\
& $\lambda$ & 2.85 & 3.333 & 8.000 & 2.000 & 8.000 & 6.667 \\
\hline
\end{tabular}

Table 2. GA parameters.

\begin{tabular}{ll}
\hline Population size $(\mathrm{N})$ & 60 \\
No. of Generation & 200 \\
Crossover probability & 0.98 \\
Mutation probability & 0.02 \\
Selection operator & Roulette Wheel \\
Crossover operator & BLX- $\alpha$ \\
Mutation operator & Polynomial mutation \\
Relative tolerance $\varepsilon$ & $10 \mathrm{E}-6$ \\
\hline
\end{tabular}

\section{Results and Discussions}

Figure 5 shows well-distributed Pareto optimal nondominated solutions obtained by the proposed algorithm after 200 generations after and before applying Local search technique.

The results declare that, implementing local search improve the solution quality for the same approach Also, for different approaches, Tables 3 and $\mathbf{4}$ show the best fuel cost and best $\mathrm{NO}_{x}$ emission obtained by proposed algorithm as compared to Nondominated Sorting Genetic Algorithm (NSGA) [14], Niched Pareto Genetic Algorithm (NPGA) [15] and Strength Pareto Evolutionary Algorithm (SPEA) [16]. It can be deduced that the proposed algorithm finds comparable minimum fuel cost and comparable minimum $\mathrm{NO}_{x}$ emission to the three evolutionary algorithms.

In this section, a compromise solution has been identified using TOPSIS technique, also the effect of changing the weights on the fuel cost and emission objectives was studied. In each case one weight is changed linearly, and the other weight was determined in such a way that $w_{1}+w_{2}=1$. In contrast, we observed the weights and the corresponding values of values of $f_{1}(\cdot)$ and $f_{2}(\cdot)$, to conclude best compromise operating point. Table 5 shows the values of the weights. The weights $\left(w_{1}, w_{2}\right)$ in six runs are shown in Table 5. Also, the best compromise solutions for different weights are shown in Figure 6.

In this subsection, a comparative study has been carried out to assess the proposed approach concerning large size problem of the Pareto-set, DM preference and computational time. On the first hand, evolutionary techniques

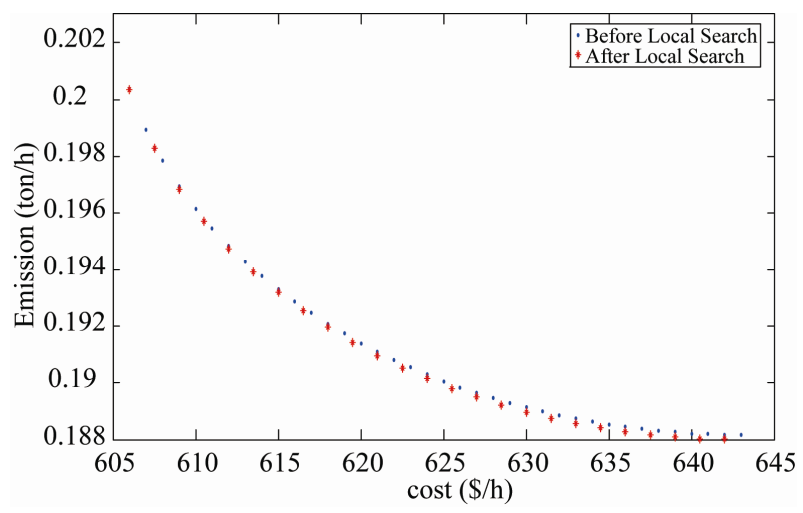

Figure 5. Pareto-optimal front of the proposed approach (Before and after applying local search).

Table 3. Best fuel cost.

\begin{tabular}{lllll}
\hline & NSGA & NPGA & SPEA & Proposed \\
\hline$P_{G 1}$ & 0.1168 & 0.1245 & 0.1086 & 0.1737 \\
$P_{G 2}$ & 0.3165 & 0.2792 & 0.3056 & 0.3568 \\
$P_{G 3}$ & 0.5441 & 0.6284 & 0.5818 & 0.5411 \\
$P_{G 4}$ & 0.9447 & 1.0264 & 0.9846 & 0.9890 \\
$P_{G 5}$ & 0.5498 & 0.4693 & 0.5288 & 0.4529 \\
$P_{G 6}$ & 0.3964 & 0.39993 & 0.3584 & 0.3705 \\
Best cost & $\mathbf{6 0 8 . 2 4 5}$ & $\mathbf{6 0 8 . 1 4 7}$ & $\mathbf{6 0 7 . 8 0 7}$ & $\mathbf{6 0 6 . 0 1 2}$ \\
Corresponding & 0.21664 & 0.22364 & 0.22015 & 0.20080 \\
Emission & & & & \\
\hline
\end{tabular}

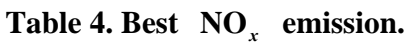

\begin{tabular}{ccccc}
\hline & NSGA & NPGA & SPEA & Proposed \\
\hline$P_{G 1}$ & 0.4113 & 0.3923 & 0.4043 & 0.3675 \\
$P_{G 2}$ & 0.4591 & 0.4700 & 0.4525 & 0.4904 \\
$P_{G 3}$ & 0.5117 & 0.5565 & 0.5525 & 0.5177 \\
$P_{G 4}$ & 0.3724 & 0.3695 & 0.4079 & 0.4512 \\
$P_{G 5}$ & 0.5810 & 0.5599 & 0.5468 & 0.5215 \\
$P_{G 6}$ & 0.5304 & 0.5163 & 0.5005 & 0.5304 \\
$\begin{array}{c}\text { Best Emission. } \\
\text { Corresponding }\end{array}$ & $\mathbf{0 . 1 9 4 3 2}$ & $\mathbf{0 . 1 9 4 2 4}$ & $\mathbf{0 . 1 9 4 2 2}$ & $\mathbf{0 . 1 8 8 0}$ \\
Cost & 647.251 & 645.984 & 642.603 & 644.5346 \\
\hline
\end{tabular}


Table 5. Different weights ( $w_{1}$ is changed linearly).

\begin{tabular}{ccc}
\hline Run & W1 & W2 \\
\hline 1 & 0.0 & 1 \\
2 & 0.1 & 0.9 \\
3 & 0.2 & 0.8 \\
4 & 0.3 & 0.7 \\
5 & 0.4 & 0.6 \\
6 & 0.5 & 0.5 \\
7 & 0.6 & 0.4 \\
8 & 0.7 & 0.3 \\
9 & 0.8 & 0.2 \\
10 & 0.9 & 0.1 \\
11 & 1.0 & 0.0 \\
\hline
\end{tabular}

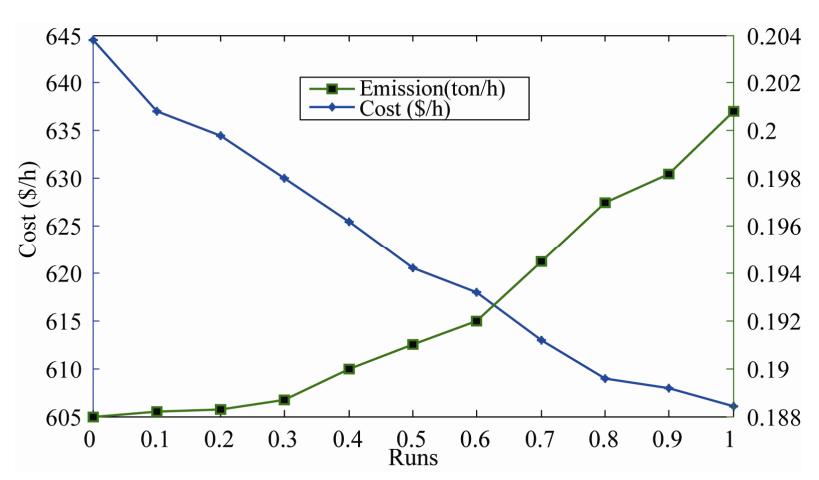

Figure 6. Best compromise solution for different weights in 11 runs of Table 5 .

suffer from the large size problem of the Pareto-set. Therefore the proposed approach has been used to reduce the Pareto-set to a manageable size. However, the goal is not only to prune a given set, but also to generate a representative subset, which maintains the characteristics of the general set and take the DM preference into consideration. Some proposed approaches have been developed using cluster analysis to reduce the size of the Pareto-set, but unfortunately it does not concern the DM preference.

On the other hand, evolutionary techniques suffer from the quality of the Pareto set. Therefore the proposed approach has been used to increase the solution quality by combing the two merits of two heuristic algorithms, genetic algorithm and local search techniques. Where, the proposed algorithm implements local search (LS) technique as neighborhood search engine such that it intends to explore the less-crowded area in the current archive to possibly obtain more nondominated solutions to improve the solution quality. TOPSIS technique has been implemented to select best compromise solution, which will satisfy the different goals to some extent by incorporating relative weights of criterion importance accordingly to DM preference. Another advantage is that the simulation results prove superiority of the proposed approach to those reported in the literature, where it completely covers and dominates all Pareto-set found by the other approaches. Finally, the reality of using the proposed approach to handle on-line problems of realistic dimensions has been approved due to small computational time.

\section{Conclusions}

The approach presented in this paper was applied to economic emission load dispatch optimization problem formulated as multiobjective optimization problem with competing fuel cost, and emission. The algorithm maintains a finite-sized archive of non-dominated solutions which gets iteratively updated in the presence of new solutions based on the concept of $\varepsilon$-dominance. Moreover, local search is employed to explore the lesscrowded area in the current archive to possibly obtain more nondominated solutions. Also to identify the best compromise solution Topsis technique was applied by incorporating relative weights of criterion importance. The following are the significant contributions of this paper:

1) The proposed technique has been effectively applied to solve the EELD considering two objectives simultaneously, with no limitation in handing more than two objectives.

2) Allowing a decision maker to control the resolution of the Pareto set approximation by choosing an appropriate $\varepsilon$ value.

3) The proposed approach has the ability to identify the best alternative from a finite set of alternatives quickly by incorporating relative weights of criterion importance.

4) The proposed approach is efficient for solving nonconvex multiobjective optimization problems where multiple Pareto-optimal solutions can be found in one simulation run.

5) Local search method is employed to explore the less-crowded area in the current archive to possibly obtain more nondominated solutions.

6) This work may be very valuable for on-line operation of power systems when environmental constraints are also need to be considered. In addition to on-line operation, this work can be a part of an off-line planning tool when there are hard limits on how much emission is acceptable by a utility over a period of a month or a year.

For further work, we intend to solve large scale EELD problem with multiple dimension in a different vision using energy market which changes the role of dis- 
patcher.

\section{Acknowledgements}

The authors are grateful to the anonymous reviewers for their valuable comments and helpful suggestions which greatly improved the paper's quality.

\section{References}

[1] S. F. Brodesky and R. W. Hahn, "Assessing the Influence of Power Pools on Emission Constrained Economic Dispatch," IEEE Transactions on Power Systems, Vol. 1, No. 1, 1986, pp. 57-62. doi:10.1109/TPWRS.1986.4334844

[2] G. P. Granelli, M. Montagna, G. L. Pasini and P. Marannino, "Emission Constrained Dynamic Dispatch," Electric Power Systems Research, Vol. 24, 1992, pp. 5664. doi:10.1016/0378-7796(92)90045-3

[3] A. Farag, S. Al-Baiyat and T. C. Cheng, "Economic Load Dispatch Multiobjective Optimization Procedures Using Linear Programming Techniques," IEEE Transactions on Power Systems, Vol. 10, No. 2, 1995, pp. 731-738. doi:10.1109/59.387910

[4] C. S. Chang, K. P. Wong and B. Fan, "Security-Constrained Multiobjective Generation Dispatch Using Bicriterion Global Optimization," IEE Proceedings Generation, Transmission \& Distribution, Vol. 142, No. 4, 1995, pp. 406-414. doi:10.1049/ip-gtd:19951806

[5] J. S. Dhillon, S. C. Parti and D. P. Kothari, "Stochastic Economic Emission Load Dispatch," Electric Power System Research, Vol. 26, 1993, pp. 179-186. doi:10.1016/0378-7796(93)90011-3

[6] J. X. Xu, C. S. Chang and X. W. Wang, "Constrained Multiobjective Global Optimization of Longitudinal Interconnected Power System by Genetic Algorithm," IEE Proceedings Generation, Transmission \& Distribution, Vol. 143, No. 5, 1996, pp. 435-446. doi:10.1049/ip-gtd:19960418

[7] J. Zahavi and L. Eisenberg, "Economic-Environmental Power Dispatch," IEEE Transactions on Systems, Man, and Cybernetics, Vol. 5, No. 5, 1985, pp. 485-489. doi:10.1109/TSMC.1975.5408370

[8] Y. T. Hsiao, H. D. Chiang, C. C. Liu and Y. L. Chen, "A Computer Package for Optimal Multi-Objective VAR Planning in Large Scale Power Systems," IEEE Transactions on Power Systems, Vol. 9, No. 2, 1994, pp. 668-676. doi:10.1109/59.317676

[9] R. Yokoyama, S. H. Bae, T. Morita and H. Sasaki, "Multiobjective Generation Dispatch Based on Probability Security Criteria," IEEE Transactions on Power Systems, Vol. 3, No. 1, 1988, pp. 317-324. doi:10.1109/59.43217

[10] B. S. Kermanshahi, Y. Wu, K. Yasuda and R. Yokoyama, "Environmental Marginal Cost Evaluation by NonInferiority Surface," IEEE Transactions on Power Systems, Vol. 5, No. 4, 1990, pp. 1151-1159. doi:10.1109/59.99365

[11] M. Azzam and A. A. Mousa, "Using Genetic Algorithm and Topsis Technique for Multiobjective Reactive Power Compensation," Electric Power Systems Research, Vol. 80 , No. 6, 2010, pp. 675-681. doi:10.1016/j.epsr.2009.10.033

[12] M. S. Osman, M. A. Abo-Sinna and A. A. Mousa, "A Solution to the Optimal Power Flow Using Genetic Algorithm," Mathematics \& Computation, Vol. 155, No. 2, 2004, pp. 391-405.

[13] M. S. Osman, M. A. Abo-Sinna and A. A. Mousa, "Epsilon-Dominance Based Multiobjective Genetic Algorithm for Economic Emission Load Dispatch Optimization Problem," Electric Power Systems Research, Vol. 79, No. 11, 2009, pp. 1561-1567. doi:10.1016/j.epsr.2009.06.003

[14] M. A. Abido, "A Novel Multiobjective Evolutionary Algorithm for Environmental/Economic Power Dispatch," Electric Power Systems Research, Vol. 65, No. 1, 2003, pp. 71-81. doi:10.1016/S0378-7796(02)00221-3

[15] M. A. Abido, "A Niched Pareto Genetic Algorithm for Multiobjective Environmental/Economic Dispatch," Electrical Power and Energy Systems, Vol. 25, No. 2, 2003, pp. 97-105. doi:10.1016/S0142-0615(02)00027-3

[16] M. A. Abido, "Environmental/Economic Power Dispatch using Multiobjective Evolutionary Algorithms," IEEE Transactions on Power Systems, Vol. 18, No. 4, 2003, pp. 1529-1537. doi:10.1109/TPWRS.2003.818693

[17] K. Deb, "Multi-Objective Optimization Using Evolutionary Algorithms," Wiley, New York, 2001.

[18] C. M .Fonseca and P. J. Fleming, "An Overview of Evolutionary Algorithms in Multiobjective Optimization," Evolutionary Computation, Vol. 3, No. 1, 1995, pp. 1-16. doi:10.1162/evco.1995.3.1.1

[19] D. L. Olson, "Comparison of Weights in TOPSIS Models," Mathematical and Computer Modelling, Vol. 40, No. 7-8, 2004, pp. 721-727. doi:10.1016/j.mcm.2004.10.003

[20] M. Laumanns, L. Thiele, K. Deb and E. Zitzler, "Archiving with Guaranteed Convergence and Diversity in Multi-Objective Optimization," GECCO 2002: Proceedings of the Genetic and Evolutionary Computation Conference, Morgan Kaufmann Publishers, New York, July 2002, pp. 439-447.

[21] D. Hazarika and P. K. Bordoloi, "Modified Loss Coefficients in the Determination of Optimum Generation Scheduling," IEE Proceedings, Vol. 138, No. 2, 1991, pp. 166-172

[22] W. Y. Ng, "Generalized Generation Distribution Factors for Power System Security Evaluations," IEEE Transactions on Power Apparatus and Systems, Vol. 100, 1981, pp. 1001-1005. doi:10.1109/TPAS.1981.316635

[23] A. A. Mousa, R. M. Rizk-Allah and W. F. A. El-Wahed, "A Hybrid Ant Colony Optimization Approach Based Local Search Scheme Formultiobjective Design Optimizations," Electric Power Systems Research, Vol. 81, No. 4, 2011, pp. 1014-1023. doi:10.1016/j.epsr.2010.12.005

[24] R. Hooke and T. A. Jeeves, "Direct Search Solution of Numerical and Statistical Problems," Journal of the $A C M$, Vol. 8, No. 2, 1961, pp. 212-229. doi: $10.1145 / 321062.321069$ 